\title{
Analyticity and higher twists
}

\author{
Oleg Teryaev ${ }^{\mathrm{a}}$ \\ ${ }^{a}$ Bogoliubov Laboratory of Theoretical Physics, Joint Institute for Nuclear Research, 141980 Dubna, Russia
}

\begin{abstract}
The representation for infinite sum of higher twists (HT) tower in DIS implied by analyticity of virtual Compton amplitude is suggested. Its simplest realization allows to describe the Bjorken sum rule at all momentum transfers. It is stressed that TMDs accommodate the infinite tower of HT similar to non-local vacuum condensates for the case of vacuum matrix element. The D-term in hadronic GPDs bears some similarity to vacuum cosmological constant. The negative sign of D-term may be understood as a similarity between inflation and annihilation via the graviton exchange.
\end{abstract}

Keywords: Higher twist, dispersion relation, resummation, condensates, cosmological constant

1. Introduction (where Bessel moments [4] should naturally appear because transverse space is 2 -dimensional) correspond to

Higher twist (HT) corrections are very important for the applications of QCD at low scales. The lower is the scale the higher twists enter into the game. Sometimes any finite number of them is insufficient, and account for infinite sums is necessary.

We address here several such cases. The first one corresponds to (spin-dependent) DIS in real-photon limit, when contact with low-energy theorems (GDH sum rules) may be achieved [1]. Originally, this was realized by matching of the HT expansion in inverse powers of $Q^{2}$ (where QCD perturbative expansion in $\log Q^{2}$ was also included [2]) and "chiral" expansion in positive powers of $Q^{2}$. Here we describe amother procedure, where HT series is represented in integral form, incorporating the analytic properties of virtual Compton amplitude. Even in its simplest version it leads to the rather accurate description of Bjorken sum rule at all $Q^{2}$.

Another situation of all-twists relevance is represented by transverse momentum dependent parton distributions (TMDs). They may be considered [3] as a (partial)sum of all HTs tower, while transverse moments

Email address: teryaev@theor.jinr.ru (Oleg Teryaev) definite twists.

The TMDs are therefore similar[5] to the non-local vacuum condensates [6] also partially resumming the local condesates of definite dimension playing the role of twist in the vacuum. This may be an example of more general similarity/universality of vacuum and hadronic matrix elements. It is interesting, that vacuum analog of so-called Polyakov-Weiss D-term [7] in generalized parton distributions (GPDs) is represented by nothing less than cosmological constant. The observed definite (negative) sign of D-term may, in turn, be interpreted as a positive "effective" cosmological constant in the annihilation channel of respective gravitational formfactor establishing the relation between inflation and annihilation.

\section{Resumming HT in spin-dependent DIS}

Let us consider as a case study the lowest non-singlet moment of spin-dependent proton and neutron structure functions $g_{1}^{p, n}$ defined as

$$
\Gamma_{1}^{p-n}\left(Q^{2}\right)=\int_{0}^{1} d x g_{1}^{p-n}\left(x, Q^{2}\right)
$$


with $x=Q^{2} / 2 M v$, the energy transfer $v$, and the nucleon mass $M$. We imply the elastic contribution at $x=1$ to be excluded, since the low- $Q^{2}$ behavior of "inelastic" $\Gamma_{1}^{p-n}\left(Q^{2}\right)$, i.e. the Bjorken Sum Rule (BSR), is constrained by the Gerasimov-Drell-Hearn (GDH) sum rule, which allows us to investigate continuation of the Bjorken integral $\Gamma_{1}^{p-n}\left(Q^{2}\right)$ to low $Q^{2}$ scales. As all the higher twists contributions are divergent when $Q^{2} \rightarrow 0$, only infinite sum may be matched to GDH value. Let us consider the series

$$
S\left(Q^{2}\right)=\sum_{1}^{\infty} a_{n}\left(\frac{M^{2}}{Q^{2}}\right)^{n} .
$$

which may correspond either to non-perturbative part (HT) of $\Gamma_{1}$ or to $I_{1}=2 M^{2} \Gamma_{1} / Q^{2}$ proportional to photoabsorption cross-sections constrained by GDHSR. By making the crucial step and representing $a_{n}$ as a moments

$$
a_{n}=\int_{-\infty}^{\infty} f(x) x^{n-1}
$$

the sum of HTs can be recasted as

$$
S\left(Q^{2}\right)=\int_{-\infty}^{\infty} d x \frac{f(x) M^{2}}{Q^{2}-x M^{2}} .
$$

Such a representation in terms of moments may be compared to the similar one when the standard leading twist partonic expression is derived. The later, besides the nice physical pictture, may be justified by correct analytical properties of virtual Compton amplitude, having $\mathrm{s}$ and $\mathrm{u}$ cuts produced by respective poles (at LO) and cuts in the partonic subprocess.

The similar arguments may be applied for HT resummation. If analytical properties of $S\left(Q^{2}\right)$ are represented by the cut residing at $Q^{2} \leq 4 m^{2}$ (m being the mass of the lightest particle in the respective channel), the integration in 3 should be limited to $\left(-\infty,-4 m^{2} / M^{2}\right)$. If the function $f(x)$ has a definite sign it leads to the alternating HT series. Moreover, even for sign-changing $f(x)$ the series will be typically alternating unless finetuning of $f$ is imposed.

It is crucially important that the leading twist contribution and respective perturbative (logarithmic) corrections has the same analytic properties, leading to the same properties of the full amplitude. This naturally selects the modified Analytic Perturbation Theory (APT) [8] which was successfully applied for the description of BSR [9]. These dtudies manifested the duality between the HT and perturbative corrrections, so that HT decreased at NLO etc. Let me conjecture here, that the "real" HT should correspond to the piece which cannot be absorbed to perturbative series due to is asymptotic nature.

One may now continue $S$ to $Q^{2}=0$ which is defined by first inverse moment

$$
S(0)=-\int_{-\infty}^{-4 m^{2} / M^{2}} d x \frac{f(x)}{x} .
$$

Its sign will typically coincide with that of the first term of the series.

The derivatives of $S$ at $Q^{2}=0$ are defined by higher inverse momentss,say

$$
S^{\prime}(0)=-\int_{-\infty}^{-4 m^{2} / M^{2}} d x \frac{f(x)}{M^{2} x^{2}} .
$$

If one neglect $m$ (which corresponds to minimal APT in the perturbative part) this integral may diverge at $x \sim 0$. This divergence may be used to cancel the infinite slope of minimal APT contribution requiring that $f(x) \sim \rho_{\text {pert }}\left(s=M^{2} x\right)$ for $x \sim 0$. This divergence is absent in the recently elaborated Massive Perturbation Theory (MPT [10], where $Q^{2} \rightarrow \tilde{Q}^{2}=Q^{2}+M_{g l}^{2}$ ) which together with the VDM form of HT contribution $M_{H T}^{2} /\left(Q^{2}+M_{H T}^{2}\right)$ lead to the reasonable description of BSR down to rather low $Q^{2}$.

Note that VDM form of HT perfectly fits to (4) with the delta-function spectral density 1 while MPT expression has also the correct analytic properties provided relevant "gluonic mass" $M_{g l} \geq \Lambda_{Q C D}$.

At the same time, the attempt to match the MPT description with GDHSR fails. The reason is obvious: the (average) slope of $\Gamma_{1}^{p-n}\left(Q^{2}\right)$ at low $Q^{2}$ is several times larger than the one following from GDHSR. This clearly supports the "two-component" approach [1] where slope is decomposed to the sum of "fast" rapidly decreasing component due to structure function $g_{2}$ and "slow" component due to structure function $g_{T}=g_{1}+g_{2}$, which for BSR provides the slope [2] enhanced by factor $\mu_{A}^{p} /\left(\left(\mu_{A}^{n}\right)^{2}-\left(\mu_{A}^{p}\right)^{2}\right) \sim 4$ determined by proton and neutron anomalous magnetic moments.

It is therefore natural to combine MPT analysis with approach [1]. The fast component contribution is controlled by Butrkardt-Cottingham sum rule free from any corrections. GDH sum rule allows to relate HT and gluon masses (appearing to be close) so that there is single free parameter remained. The one-parameter fits ${ }^{2}$

\footnotetext{
${ }^{1}$ It is interesting that similar VDM form was discussed at this conference also in the talks of Ya.Klopot for pion-photon transition formfactor [11] (where it is related to axial anomaly) and A. Aleksejevs for pion formfactor

${ }^{2}$ I am grateful to I.Gabdrakhmanov and V. Khandramai for the help in numerical calculations.
} 


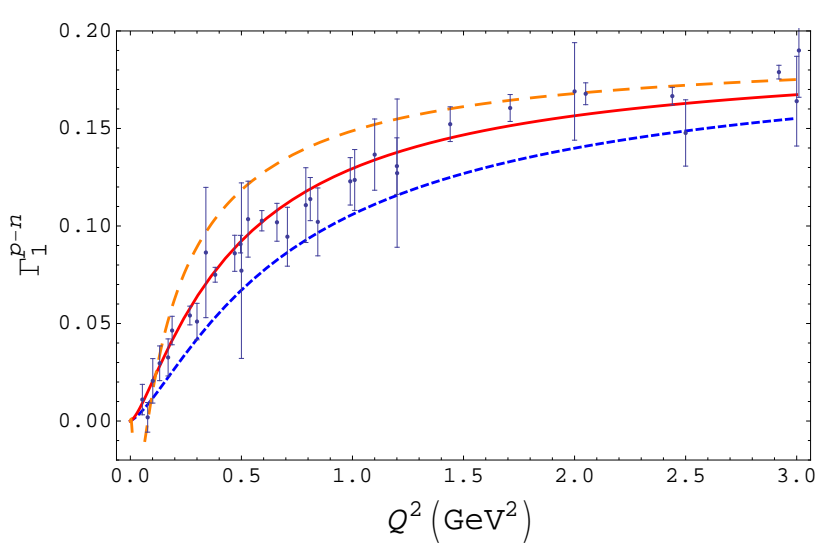

Figure 1. BSR at LO (dotted blue curve), NLO (solid red curve), $\mathrm{NNLO}$ (dashed yellow curve) compared with experimental data.

lead to the reasonable description of the data with the quality increasing with taking into account NLO MPT [10] and modifications of spectral density (4),

It is well known that modified scaling variables provide the way of partial HT resummation. This corresponds to famous Nachtmann scaling variable and another one $\hat{x}=\tilde{Q}^{2} / 2 M v$, closely related to MPT and used at low $x$ region. The advantage of the last variable for BSR studies is that it is larger than $x_{B}$ so that $\left.g_{(} \hat{x}\right)<g_{1}\left(x_{B}\right)$ and one may hope to describe [12] the decrease of $\Gamma_{1}^{p-n}\left(Q^{2}\right)$ at small $Q^{2}$ required by the finiteness of photoabsorption cross-section. However, for larger $x_{B}$ the spectral condition for $\hat{x}$ may be violated, so that $\hat{x} \geq 1$. To avoid that, one may introduce another variable, using the representation $x_{B}=Q^{2} /\left(Q^{2}+W^{2}\right)$ and performing the same change $Q \rightarrow \tilde{Q}$ :

$$
\tilde{x}=x_{B} \frac{1+a}{1+a x_{B}}, a=\frac{M_{g l}^{2}}{Q^{2}} .
$$

The first moment may be now presented as

$$
\tilde{\Gamma}_{1}\left(Q^{2}\right)=\int_{0}^{1} g_{1}(\tilde{x}) d x=(a+1) \int_{0}^{1} \frac{g_{1}(z) d z}{(1+a(1-z))^{2}}
$$

While asymptotically this result reproduces the standard partonic expression, at smail $Q^{2}$ one get

$$
\tilde{\Gamma}_{1}\left(Q^{2}\right) \rightarrow \frac{Q^{2}}{M_{g l}^{2}} \int_{0}^{1} \frac{g_{1}(z) d z}{(1-z)^{2}},
$$

and the slope at the origin related to GDHSR is defined by the second inverse moment. Note that the contribution of large $\mathrm{x}$ region is enhanced. The available parameterizations of spin-dependent parton distributions seem to provide a rather reasonable of data at low $Q^{2}[12]$.

\section{Hadronic vs vacuum matrix elements}

\subsection{Transverse Momentum Dependent Distributions and vacuum condensates}

The TMDs can be conveniently defined[5] in coordinate (impact parameter) space. The representative case is Boer-Mulders function (in order to avoid consideration of gauge links/gluonic poles one may consider[5] Collins fragmentation function having the same Lorentz structure) when transverse coordinate is selected by the chiral-odd Dirac structure

$$
\left\langle P\left|\bar{\psi}(0) \sigma^{\mu v} \psi(z)\right| P\right\rangle=M\left(P^{\mu} z^{v}-P^{v} z^{\mu}\right) I\left(z \cdot P, z^{2}\right)
$$

Here the $z^{2}$-dependence corresponds to $k_{T}$ dependence in momentum space and contains all twists. The definite twists may be extracted by the expansion in Taylor series

$$
I\left(z \cdot P, z^{2}\right)=\left.\sum_{n=0}^{\infty} \frac{\partial^{n}}{n ! \partial z^{2 n}} I\left(z \cdot P, z^{2}\right)\right|_{z^{2}=0},
$$

which in the momentum space corresponds to the transverse moments of TMD. Note that the lowest twist is 3, which is seen from the appearance of factor $M$ in the r.h.s. of (10). In the momentum space this factor is shifted to the denominator, as the transverse moment is taken over $d k_{T}^{2} / M^{2}$ [5].

Note that the expansion in $z^{2}$ may be performed only after the subtraction of the singular terms in $z^{2}$, which in the collinear factorization are absorbed to coefficient function. For TMDs they constitute the power-like tail, after subtraction of which all the transverse moments became finite, indicating the Gaussian distributions.

It is interesting that the appearance of such a tail was recently deduced [13] from the causality arguments, being as it is well known also the origin of analytic properties discussed above.

The described situation is rather similar to non-local vacuum condensates (see [6] and Ref. therein) where one is dealing with vacuum matrix element

$$
\langle 0|\bar{\psi}(0) \psi(z)| 0\rangle=\langle 0|\bar{\psi}(0) \psi(0)| 0\rangle F\left(z^{2}\right) .
$$

The Taylor expansion of F selects the local condensates of definite dimension, corresponding to the moments of suitably chosen Fourier transformed function being the complete analog of TMD. Note that subtraction of singular terms is performed here by subtraction of the perturbative contribution corresponding to quark propagator.

Generally, the hadronic matrix elements differ from the vacuum ones by the presence of essentially pseudoEuclidian hadron momentum. At the same time, the Euclidian transverse dynamics may be more vacuum-like. 


\subsection{D-term and cosmological constant}

Let us discuss one more interesting example of interplay between hadronic and vacuum matrix elements. It corresponds to so-called Polyakov-Weiss D-term [7] (appearing in analyticity based analysis as a subtraction constant [14]) whose moment is related to quadrupole gravitational formfactor transverse coordinate is selected by the chiral-odd Dirac structure

$$
\left\langle P+q / 2\left|T^{\mu v}\right| P-q / 2\right\rangle=C\left(q^{2}\right)\left(g^{\mu v} q^{2}-q^{\mu} q^{v}\right)+\ldots
$$

where gravitoelectric and gravitomagnetic formfactors [17] are dropped. C has definite (positive) sign in all the known cases including hadrons [7](where also general stability arguments are discussed), photons [15], Q-balls [16].

For vacuum matrix element one has the famous cosmological constant

$$
\left\langle 0\left|T^{\mu \nu}\right| 0\right\rangle=\Lambda g^{\mu \nu}
$$

One may relate this matrix element in 2-dimensional transverse space orthogonal to $\mathrm{P}$ and $\mathrm{q}$, so that effective 2-dimensional cosmological constant is

$$
\Lambda=C\left(q^{2}\right) q^{2}
$$

The positive $\mathrm{C}$ leads to negative cosmological constant in the scattering process and to positive one in the annihilation process. There seems to be some relation between annihilation and inflation! It may not be so surprising due to known similarity 3 between inflation and Schwinger pair production in the electric field.

It is of course very interesting whether real cosmological constant in our Universe may be understood as emerging from annihilation at extra dimensions. Qualitatively this is similar to brane cosmology, and one should stress that in the suggested scenario Big Bang is due to one-graviton annihilation. The specification of extra-dimensional states providing the cosmological constant of mass dimension 4 remains to be investigated,

\section{Conclusions}

The analyticity property continues to play the major role in developing of QCD approaches to low scale processes, being of most experimental interest. It allows one to justify the representation of infinite sums of higher twists contributions, providing, in particular, the

\footnotetext{
${ }^{3}$ I am indebted to A.A. Starobinsky for this comment.
}

accurate description of Bjorken Sum Rule data at low $Q^{2}$. Another method of higher twists partial resummation is provided by modified scaling variables, sometimes allowing to describe the real photon limit of DIS.

The infinite series of higher twists are required to transverse momentum dependent parton distributions, having deep similarity to non-local vacuum condensates.

The vacuum/hadron matrix elements similarity allows to describe one-graviton annihilation as effective 2-dimensional cosmological constant, and this may be geberalized to describe in a similar way cosmological constant in our Universe as emerging from one-graviton annihilation at extra dimension, which is the picture of a Big Bang in such a case.

I am grateful to Organizers for warm hospitality at HIgh Tatras and to Participants for many exciting discussions. This work is supported in parst by RFBR grants 11-01-00182, 12-02-00613 and 13-02-01060.

\section{References}

[1] J. Soffer and O. Teryaev, Phys. Rev. Lett. 70 (1993) 3373; Phys. Rev. D 51 (1995) 25 |hep-ph/9405228].

[2] J. Soffer and O. Teryaev, Phys. Rev. D 70 (2004) 116004 |hepph/0410228

[3] P. G. Ratcliffe and O. V. Teryaev, hep-ph/0703293, Mod. Phys. Lett. A 24 (2009) 2984 |arXiv:0910.5348 [hep-ph]].

[4] D. Boer, L. Gamberg, B. Musch and A. Prokudin, JHEP 1110 (2011) 021 arXiv:1107.5294 [hep-ph]].

[5] O. V. Teryaev, Phys. Part. Nucl. 35 (2004) S24.

[6] A. P. Bakulev and A. V. Radyushkin, Phys. Lett. B 271 (1991) 223.

[7] K. Goeke, M. V. Polyakov and M. Vanderhaeghen, Prog. Part. Nucl. Phys. 47 (2001) 401 [hep-ph/0106012].

[8] D. V. Shirkov and I. L. Solovtsov, Phys. Rev. Lett. 79 (1997) 1209 |hep-ph/9704333|;Theor. Math. Phys. 150 (2007) 132 |hep-ph/0611229].

[9] R. S. Pasechnik, D. V. Shirkov and O. V. Teryaev, Phys. Rev. D 78 (2008) 071902 [arXiv:0808.0066 [hep-ph]]; V. L. Khandramai, R. S. Pasechnik, D. V. Shirkov, O. P. Solovtsova and O. V. Teryaev, Phys. Lett. B 706 (2012) 340 |arXiv:1106.6352 [hep-ph]].

[10] D. V. Shirkov, Phys. Part. Nucl. Lett. 10 (2013) 186 arXiv:1208.2103 [hep-th]].

[11] Y. Klopot, A. Oganesian and O. Teryaev, arXiv:1308.5184 [hep$\mathrm{ph}$.

[12] D. Kotlorz and O. Teryaev, work in progress.

[13] A. V. Efremov and A. A. Vladimirov, arXiv:1306.3929 [hep$\mathrm{ph}$.

[14] O. V. Teryaev, hep-ph/0510031 I. V. Anikin and O. V. Teryaev, Phys. Rev. D 76 (2007) 056007 [arXiv:0704.2185[hep-ph]]

[15] I. R. Gabdrakhmanov and O. V. Teryaev, Phys. Lett. B 716 (2012) 417 [arXiv:1204.6471 [hep-ph]].

[16] M. Mai and P. Schweitzer, Phys. Rev. D 86 (2012) 076001 arXiv:1206.2632 [hep-ph]];ibid., 096002 arXiv:1206.2930 [hep-ph]].

[17] O. V. Teryaev, hep-ph/9904376 A. J. Silenko and O. V. Teryaev, Phys. Rev. D 71 (2005) 064016 |gr-qc/0407015|. 
Y. N. Obukhov, A. J. Silenko and O. V. Teryaev, Phys. Rev.

D 80 (2009) 064044 arXiv:0907.4367 [gr-qc]]; O. V. Se-

lyugin and O. V. Teryaev, Phys. Rev. D 79 (2009) 033003

[arXiv:0901.1786 [hep-ph]]. 